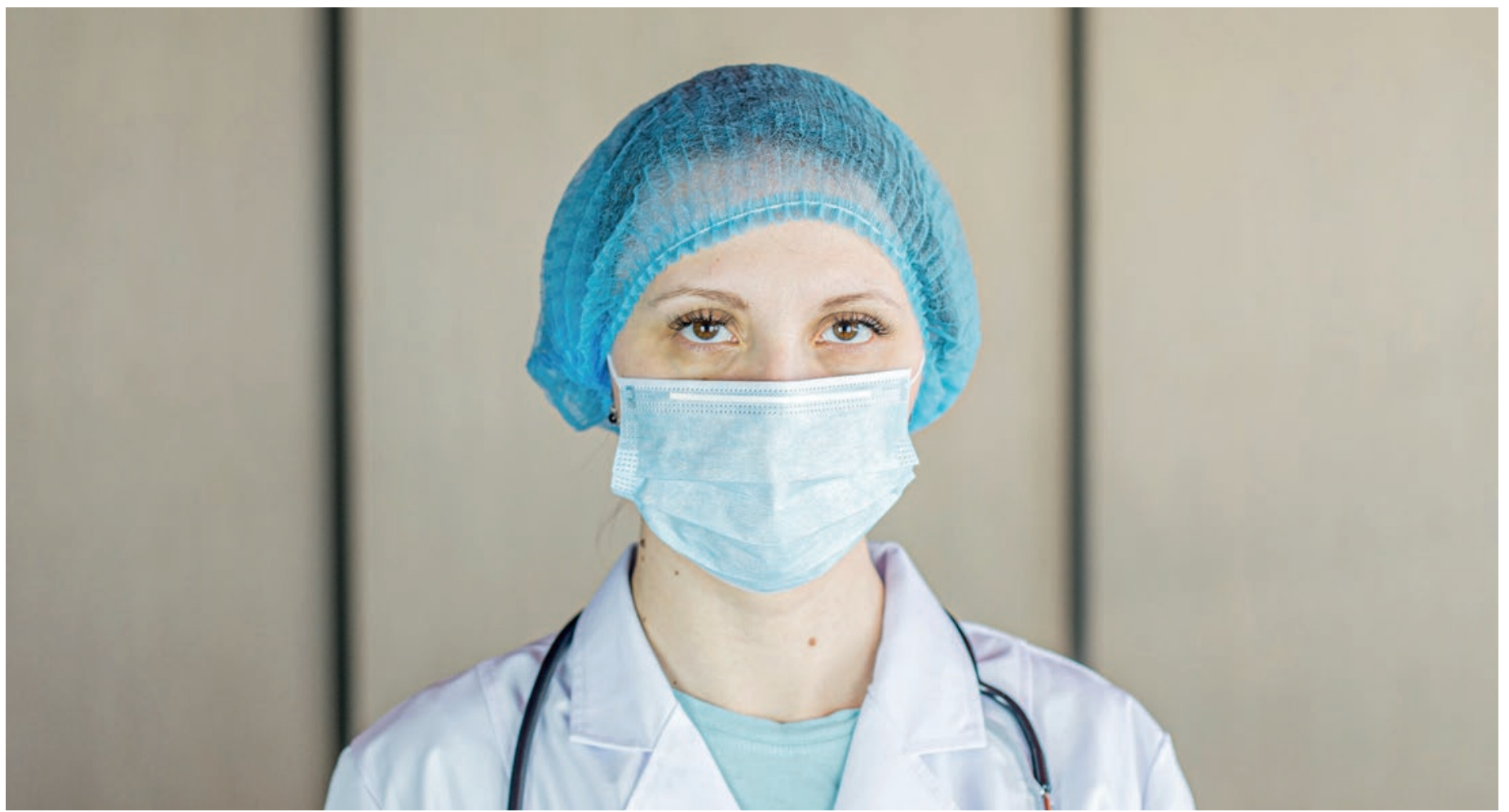

\title{
Als Helden der Pandemie beklatscht - dann im Stich gelassen
}

\section{Marcus M. Maassen}

Prof. Dr. med., Präsident PULSUS

Pflegekräfte und Ärzteschaft unterstützen den Staat und die Gesellschaft bei der Bewältigung der Corona-Pandemie massiv. Andererseits werden die Ärzte mit einem Gesundheitswesen konfrontiert, das durchdrungen ist vom Geist der Planwirtschaft und des Interventionismus. Die Strategie "Gesundheit 2030» des Bundesrats visiert ein Globalbudget an und fördert Rationierungen. Das ist nicht fair.

Wer sich mit den Auswirkungen der Pandemie auf unsere Gesellschaft beschäftigt, erlebt die Bedeutung der Ärzte und Pflegekräfte hautnah und beobachtet einen bis zur körperlichen und psychischen Erschöpfung gehenden Einsatz auf den Intensivpflegestationen. Ebenso verdient das Engagement der Hausärzte und Fachärzte Respekt, die trotz Mangel an Desinfektionsmittel und Schutzkleidung sowie einem Behandlungsverbot aller nicht dringend angezeigten Behandlungen und Eingriffe ihre Praxen im März und April 2020 offenhielten. Dieses Behandlungsverbot führte zusammen mit den verschärften Hygieneregeln sowie der Notwendigkeit von social distancing im vergangenen Jahr zu einem Umsatzrückgang von mindestens 5\%. Gemäss FMH verursacht ein Umsatzrückgang von 5\% einen Erwerbsausfall von 13 bis 17\%.

Die Pandemie hat gezeigt, dass eine gute Gesundheitsversorgung für die Bevölkerung Priorität hat.

Sehr wichtig ist die Bedeutung der Spitalärzte und Pflegekräfte, die auf den Notfallstationen und im Rettungswesen viele Menschenleben gerettet haben. Das unermüdliche Engagement der Ärzte und Pflegekräfte, sich auch nach einer anstrengenden Arbeitswoche 
zusätzlich am Wochenende für den Dienst in einem kantonalen Impfzentrum einteilen zu lassen, hat die schnelle Umsetzung der Impfstrategie der Kantone wesentlich unterstützt. Jetzt, nachdem die dritte Welle gebrochen ist und die Inzidenzen im Wochenvergleich rückgängig sind, sei das Zwischenresümee erlaubt,

Das Behandlungsverbot während der Pandemie, die verschärften Hygieneregeln und die Notwendigkeit von social distancing führten im vergangenen Jahr zu einem Umsatzrückgang von $5 \%$.

dass Pflegekräfte und Ärzteschaft den Staat und die Gesellschaft bei der Bewältigung dieser historischen Aufgabe in der Pandemie massiv unterstützen.

Die Pandemie hat gezeigt, dass eine gute Gesundheitsversorgung für die Bevölkerung Priorität hat. Eine aktuelle Umfrage des Forschungsinstitutes gfs.bern (Gesellschaft für Sozialforschung) ergab, dass die Mehrheit der Befragten für die Deckung sämtlicher Leistungen plädiert. Die Mehrheit will Zugang zu Medikamenten, freie Arztwahl und mehr Leistungen auch im hohen Alter. Für die Bevölkerungsmehrheit ergibt dies eine Verschiebung der Prioritäten: weg von den Kosten und hin zu einer umfassenderen Versorgung, mit Zugang zu den Medikamenten und einer weiterhin freien Arztwahl.

\section{Planwirtschaft und Interventionismus im Gesundheitswesen}

Hat der Staat die Ärzteschaft unterstützt? Einerseits hat die Bereitstellung der COVID-19-Darlehen viele Unternehmen und Praxen vor dem drohenden Ruin gerettet. Andererseits werden die Ärzte mit einem Gesundheitswesen konfrontiert und von diesem behindert, das durchdrungen ist vom Geist der Planwirtschaft und des Interventionismus, statt auf ärztliche Eigenverantwortung zu setzen. Ein zukunftsfähiges Gesundheitssystem kann nur dann einen attraktiven Mehrwert für die Bevölkerung leisten, wenn dezentrale Strukturen, die sich über Jahrzehnte bewährt haben, weitergeführt werden. Heute ist es aber für viele Ärzte faktisch nicht mehr möglich, einen Nachfolger für ihre Praxen zu finden. Ursache sind auch die Ertragsaussichten einer Praxistätigkeit, die nach den seit 2014 durchgeführten zwei Kürzungen in der Gebührenordnung TARMED eingebrochen sind. Weitere Einschnitte der Vergütung sind bei der Einführung von Pauschalen geplant. Unverständlich und nicht nachvollziehbar ist der Entscheid des Bundesrates, die Genehmigung des TARDOC zu verschieben.
Ebenso umstritten wie die Einführung von sanktionierbaren Mengenbegrenzungen im Rahmen einer KVG-Revision ist die auf der politischen Agenda stehende Einführung eines Globalbudgets. Dieses wird zu einem Abbau von Kapazitäten und zweifelsfrei zu längeren Wartezeiten auf medizinische Behandlungen führen. Warum sollten diese Auswirkungen eines Globalbudgets gerade in der Schweiz nicht eintreten, wenn doch die Erfahrungen in allen anderen Ländern (zum Beispiel Deutschland, Grossbritannien, Spanien, Italien) mit einem Globalbudget zu viel längeren Wartezeiten auf medizinische Leistungen und einer ZweiKlassen-Medizin geführt haben.

\section{Strategie Gesundheit 2030 visiert Globalbudget an}

Man darf sich keinen Illusionen hingeben: Auch in der Zukunft werden wir in der Schweiz einen Mangel an qualifizierten Gesundheitsfachpersonen haben. Der Zugang zum Gesundheitssystem muss auch für Menschen mit bescheidenem sozioökonomischem Status gewährleistet sein. Diese Probleme lassen sich nicht mit einem ausufernden Staatsinterventionismus lösen. Die von Herrn Bundesrat Berset vorgeschlagenen «Zielvorgaben» zielen auf ein Globalbudget hin. Zielvorgaben verfehlen aber hier das Ziel. Darauf weist Urs Stoffel mit Recht in der Schweizerischen Ärztezeitung 2021; 102(22):73 hin. Mit politischem Kalkül wurden die Massnahmen «Zielvorgaben» mittlerweile aus dem Kostendämpfungspaket 2 herausgelöst und zum indirekten Gegenvorschlag der Kostenbremse-Initiative erklärt. Definitiv wird durch diese Massnahmen das neue Zeitalter des Globalbudgets eingeläutet.

Zwar wird in der Agenda 2030 vollmundig behauptet, dass die Strategie internationale gesundheitspolitische Entwicklungen und Best Practices nutzt, doch

\section{Ein Globalbudget wird zu einem Abbau von}

Kapazitäten und zweifelsfrei zu längeren Wartezeiten auf medizinische Behandlungen führen.

werden die negativen internationalen Erfahrungen mit dem Globalbudget ausgeblendet. Im internationalen Vergleich führt ein Globalbudget zu Rationierungen.

So werden beispielsweise Knie- und Hüftprothesenoperationen in Grossbritannien erst dann durchgeführt, wenn der Patient so starke Schmerzen hat, dass er nachts nicht mehr schlafen kann (dailymail.co.uk vom 27.1.2017). Dies ist eine implizite Auswirkung eines Globalbudgets, was zu Rationierungen medizinischer Leistungen geführt hat. 
Die Folge ist, dass ein medizinischer Tourismus besteht und die wohlhabenden Patienten aus Grossbritannien sich in anderen Ländern operieren lassen. Es besteht die Gefahr, dass die Schweiz die Fehler wiederholt, die in anderen Ländern nachweislich zu einer Verschlechterung der medizinischen Versorgung geführt haben. Mit dem Weg zur Planwirtschaft und zum Interventionismus wird der Schweizer Bevölkerung der Zugang zu einer qualitativ hochstehenden Gesundheitsversorgung in den kommenden Jahren erschwert.

\section{Globalbudget fördert Rationierungen}

Die von Gesundheitsminister Alain Berset vorgeschlagenen Sparmassnahmen mit Einführung degressiver Tarife werden jedoch zu einem Globalbudget mit Rationierungen führen. Würden die Vergütungen für medizinische Leistungen mengenabhängig sinken und hätten Ärzte und Therapeuten nachträglich finanzielle Sanktionen für notwendige Leistungen zu erwarten, so würden medizinisch notwendige Leistungen nicht mehr erbracht werden. Dieses ist eine nicht verfassungskonforme Verletzung des Versicherungsprinzips,

\section{Das Wichtigste in Kürze}

- Die Mehrheit der Bevölkerung will Zugang zu Medikamenten, freie Arztwahl und mehr Leistungen auch im hohen Alter. Dies ergibt eine Verschiebung der Prioritäten weg von den Kosten, hin zu einer umfassenderen Versorgung.

- Schweizer Ärztinnen und Ärzte werden allerdings mit einem Gesundheitswesen konfrontiert und von diesem behindert, das durchdrungen ist vom Geist der Planwirtschaft und des Interventionismus. Die negativen internationalen Erfahrungen mit dem Globalbudget werden von der Politik ausgeblendet. Im internationalen Vergleich führt ein Globalbudget zu Rationierungen.

- Hätten Ärzte und Therapeuten nachträglich finanzielle Sanktionen für notwendige Leistungen zu erwarten, so würden medizinisch notwendige Leistungen nicht mehr erbracht werden. Dieses ist eine nicht verfassungskonforme Verletzung des Versicherungsprinzips, wonach jeder Bürger die notwendige medizinische Leistung erhalten muss. wonach jeder Bürger die notwendige medizinische Leistung erhalten muss.

Es entbehrt nicht einer gewissen Pikanterie, dass ausgerechnet die in der Pandemie oft am Limit arbeitenden, systemrelevanten Ärzte und Pflegekräfte im

Es besteht die Gefahr, dass die Schweiz die Fehler wiederholt, die in anderen Ländern nachweislich zu einer Verschlechterung der medizinischen Versorgung geführt haben.

ambulanten und stationären Sektor zuerst als Helden der Pandemie gefeiert werden, gleichzeitig durch die geplante Einführung eines Globalbudgets vom Bundesrat bestraft werden.

PULSUS hat erhebliche, auch rechtliche Bedenken gegen Globalbudgets und lehnt die Einführung eines Globalbudgets vehement ab. Nach Abflachen der dritten Welle der Pandemie fühlen sich Ärzte und Pflegekräfte von der Politik im Stich gelassen.

\section{Bildnachweis}

Bermix Studio / Unsplash 